\title{
Hemoglobin A1c modifies the association between triglyceride and time in hypoglycemia determined by flash glucose monitoring in adults with type 1 diabetes: implications for individualized therapy and decision-making
}

\author{
Yiwen Liu ${ }^{1}$, Jie Yu ${ }^{1}$, Chifa $\mathrm{Ma}^{1}$, Shuli $\mathrm{He}^{2}$, Fan Ping ${ }^{1}$, Huabing Zhang ${ }^{1}$, Wei Li $^{1}$, Lingling Xu ${ }^{1}$, \\ Xinhua Xiao ${ }^{1}$, Yuxiu $\mathrm{Li}^{1}$ \\ ${ }^{1}$ Department of Endocrinology, Key Laboratory of Endocrinology, Ministry of Health, Peking Union Medical College Hospital, Peking Union Medical \\ College, Chinese Academy of Medical Sciences, Beijing, China; ${ }^{2}$ Department of Nutrition, Peking Union Medical College Hospital, Beijing, China \\ Contributions: (I) Conception and design: Y Liu, J Yu, Y Li, X Xiao; (II) Administrative support: C Ma, F Ping, H Zhang, W Li, L Xu; (III) Provision \\ of study materials or patients: Y Liu, J Yu; (IV) Collection and assembly of data: Y Liu, J Yu, S He; (V) Data analysis and interpretation: Y Liu; (VI) \\ Manuscript writing: All authors; (VII) Final approval of manuscript: All authors. \\ Correspondence to: Yuxiu Li. Department of Endocrinology, Key Laboratory of Endocrinology, Ministry of Health, Peking Union Medical College \\ Hospital, Peking Union Medical College, Chinese Academy of Medical Sciences, Beijing, China. Email: liyuxiu@medmail.com.cn.
}

Background: We aimed to investigate the associations of flash glucose monitoring (FGM)-derived metrics with lipid profiles and identify potential modifiers of these associations among adults with type 1 diabetes (T1D).

Methods: A cross-sectional study was conducted among 108 Chinese adults with T1D who used FGM for 14 consecutive days. The relationship between FGM-derived metrics and lipid variables and potential modifiers were identified using interaction and subgroup analysis.

Results: Serum triglyceride level inversely correlated with time below range (glucose $<3.9 \mathrm{mmol} / \mathrm{L}$ ) and time in range (glucose $3.9-10.0 \mathrm{mmol} / \mathrm{L}$ ) and positively correlated with time above range (glucose $>10.0 \mathrm{mmol} / \mathrm{L}$ ) (Spearman's $\mathrm{r}=-0.34,-0.25,0.34$, respectively, all $\mathrm{P}<0.01$ ). Additionally, triglyceride levels had positive correlation with absolute measures of glycemic variability (GV) but not with the coefficient of variation for glucose (Spearman's $\mathrm{r}=0.12, \mathrm{P}>0.05$ ), a relative measure. Multivariate linear regression analysis adjusting for potential confounders including gender, age, disease duration, body mass index (BMI), daily insulin dose, fasting C-peptide, and dyslipidemia medication use showed that higher triglyceride level independently predicted decrease in time below range and time in range and increase in time above range (all $\mathrm{P}<0.01)$. Furthermore, interaction analysis found that the interaction between HbA1c and triglyceride was significant in the time below range $(\mathrm{P}$ for interaction $=0.034)$. The association between triglyceride and time below range differed substantially after stratification by HbA1c, which was significant in those with HbA1c $<7.0 \%$ whereas inconsequential among those with HbA1c $\geq 7.0 \%$. In those with HbA1c $<7.0 \%(n=44)$, the area under receiver operating characteristic curve of triglyceride predicting achievement of targets of time below range $(<4 \%)$ was 0.856 (95\% confidence interval $0.688-1.000, \mathrm{P}=0.042)$ with an optimal cutoff value of $0.50 \mathrm{mmol} / \mathrm{L}$ (sensitivity $100 \%$, specificity $66.7 \%$, positive predictive value $94.4 \%$ ).

Conclusions: In adults with T1D, HbA1c may be a potential modifier of the association between triglyceride and time below range, suggesting it might be necessary for those with $\mathrm{HbA1c}<7.0 \%$ accompanied by lower triglyceride levels to set a less intensive glycemic target to minimize risk of hypoglycemia.

Keywords: Flash glucose monitoring (FGM); glucose variability; time in ranges; triglyceride; type 1 diabetes (T1D)

Submitted Sep 09, 2020. Accepted for publication Dec 04, 2020.

doi: 10.21037/atm-20-6344

View this article at: http://dx.doi.org/10.21037/atm-20-6344 


\section{Introduction}

Type 1 diabetes (T1D) is characterized by absolute deficiency of insulin secretion caused by immunemediated pancreatic $\beta$-cell destruction, resulting in lifelong dependence on exogenous insulin treatments. Drastic glycemic variability (GV) and recurrent hypoglycemia, a hallmark of $\mathrm{T} 1 \mathrm{D}$, pose an immense challenge in maintaining optimal glycemic control in T1D. Moreover, both have been associated with long-term diabetes complications as well as mortality $(1,2)$, apart from the wellestablished risk factor hemoglobin A1c (HbA1c). Although, HbA1c represents overall glycemic control over a period of 2 to 3 months, it cannot provide specific information about the amplitude of $\mathrm{GV}$ and the occurrence of acute glycemic events including hypoglycemia and hyperglycemia. HbA1c thus fails to optimize individualized therapy and decisionmaking in diabetes management (2-6), especially for T1D. Even though HbA1c is the most canonical standard for glycemic control, other metrics such as GV, glucose patterns, frequency of hypoglycemia, and time in target are equally important components of glycemic management of T1D, which could facilitate individualized therapy and target setting (6). Continuous glucose monitoring (CGM) has garnered increasing attention for its capability in providing comprehensive information on $\mathrm{GV}$, time in target, glucose patterns, as well as frequency and severity of hypoglycemia and hyperglycemia. More recently, there has been a spike in interest in flash glucose monitoring (FGM) in routine clinical practice for its easy access to glucose readings without need for calibration with finger prick glucose tests and non-inferiority to other well-established CGM sensors in terms of device accuracy (7). Mounting evidence from randomized clinical trials and real-world studies demonstrated that patients with T1D benefited from FGM use in various aspects including improvement of HbA1c (8), reduction of GV and hypoglycemia (9), as well as enhancement in treatment satisfaction and mental well-being (10). Similar results were seen among individuals with type 2 diabetes, showing that FGM could lower HbA1c and improve treatment satisfaction without increasing hypoglycemia (11). With substantial rise in FGM use in clinical settings, GV and time in range metrics derived from FGM have exerted increasing impact on individualized therapy and decision-making in T1D; however, this effect has been largely undefined and warrants further investigation. It has been well-established that lipid profiles are closely related to glycemic control determined by HbA1c in T1D $(12,13)$. Nevertheless, it remains unexplored how $\mathrm{GV}$ and time in range metrics derived from FGM are associated with alteration in lipids in T1D and whether there exist variables that modify these associations. Therefore, we sought to conduct a cross-sectional study in adults with T1D to investigate the associations of GV and time in range metrics derived from FGM with lipid profiles and identify the potential modifiers of these associations. We present the following article in accordance with the STROBE reporting checklist (available at http://dx.doi. org/10.21037/atm-20-6344).

\section{Methods}

\section{Study population}

We performed a cross-sectional study among Chinese adults with T1D who visited the endocrinology department of Peking Union Medical College Hospital from November 2018 to June 2019. The inclusion criteria were as follows: (I) age $\geq 18$ years; (II) clinical diagnosis of T1D, which was defined as diabetes with fasting C-peptide level $\leq 0.8 \mathrm{ng} / \mathrm{ml}$ at diabetes onset; (III) body mass index (BMI) $\leq 35 \mathrm{~kg} / \mathrm{m}^{2}$; (IV) continuous insulin therapy for at least 3 months prior to enrollment in the study, which included insulin injections at least twice per day. The exclusion criteria included: (I) previous use of any kind of CGM system 3 months prior to enrollment in the study; (II) female patients who were pregnant or breastfeeding; (III) patients receiving systemic glucocorticoid therapy for any reason; (IV) history of diabetic ketoacidosis or hyperglycemic hyperosmolar syndrome in the past 3 months. The study was conducted in accordance with the Declaration of Helsinki (as revised in 2013). The study was conducted under a protocol approved by the Ethics Committee of Peking Union Medical College Hospital (Approval No. B275). All participants voluntarily signed the written informed consent form. A total of 114 adults were enrolled in the study. All participants were assigned to wear FGM sensors (Freestyle Libre, Abbott, Witney, UK) for 14 consecutive days. The participants were blinded to their FGM data to avoid any bias with respect to GV from the participants. To guarantee the accuracy and reliability of the data, those with more than $70 \%$ FGM data available within the 14-day period were finally included in the study (14). Consequently, data of 108 individuals were included in the 
final analyses.

\section{Laboratory analysis}

The FGM sensors were inserted on day 0 and removed on day 14 at the same time of day. The laboratory analysis of blood samples was performed at baseline (study day 0 ). After an overnight fast for at least 10 hours, venous blood samples were drawn in the morning. The level of plasma glucose was determined by the glucose oxidase assay, and the level of C-peptide was detected using chemiluminescence immunoassay with a detection limit of $0.01 \mathrm{ng} / \mathrm{mL}$. HbA1c was assessed using a high-performance liquid chromatography assay. Total cholesterol (TC), total triglyceride (TG), high-density lipoprotein cholesterol (HDL-C), and low-density lipoprotein cholesterol (LDL-C) as well as level of uric acid (UA) were measured using an automatic analyzer.

\section{Analysis of data from FGM and calculations of FGM- derived metrics}

The data recorded on the day the glucose sensor for FGM was inserted were excluded to avoid any bias due to insufficient glucose stabilization between the sensor and the interstitial fluid during the first 24 hours after insertion of the sensor. Thirteen validated $24-\mathrm{h}$ glycemic profiles (calculated from glucose readings obtained at 15min intervals), were used for calculations of FGM-derived metrics, including mean of glucose (Mean), coefficient of variation for glucose (CV) (15), standard deviation of glucose (SD), mean amplitude of glucose excursion (MAGE) (16), mean of daily differences (MODD) (17), interquartile range of glucose (IQR), time below range (glucose $<3.9 \mathrm{mmol} / \mathrm{L}$ ) (TBR), time in range (glucose $3.9-10.0 \mathrm{mmol} / \mathrm{L}$ ) (TIR), time above range (glucose $>10.0 \mathrm{mmol} / \mathrm{L}$ ) (TAR).

\section{Statistical analyses}

Statistical analyses were implemented using RStudio version 1.2.1335 (https://www.rstudio.com). Continuous data were presented as mean $\pm \mathrm{SD}$ (normal distribution) or median (IQR) (skewed distribution), whereas categorical data were presented as percentages. Spearman's correlation analysis was performed to explore bivariate correlations. Stepwise multivariate linear regression analyses were conducted to determine the independent predictors for the FGM-derived variables after adjusting for potential confounders. To determine performances of the lipid variables for predicting the FGM-derived variables, receiver operating characteristic (ROC) curves were established. Statistical significance was set at a two-sided $\mathrm{P}$ value $<0.05$.

\section{Results}

\section{Characteristics of the study population}

As presented in Table 1, data from a total of 108 participants (39 males and 79 females) aged between 18 and 77 years were included in the final analyses. The median of disease duration and HbA1c was 7.7 years and $7.4 \%$, respectively. The fasting C-peptide level of the participants was quite low with a median of $0.01 \mathrm{ng} / \mathrm{mL}$. The lipid profiles were within normal ranges in most participants, and the percentage of dyslipidemia medication use was as low as $8.3 \%$ (8/108), probably because of the relatively young age and normal-to-low BMI of the participants. The average CV was $40 \%$, indicating that more than half of the patients suffered from frequent hypoglycemic episodes because a $\mathrm{CV}$ higher than $36 \%$ suggests labile diabetes. According to the recommendations from the international consensus on time in range (14), the targets for glycemic control in adults with T1D were as follows: TIR $>70 \%$, TBR $<4 \%$, and TAR $<25 \%$. The average TIR was $56.68 \%$, whereas the median of TBR and TAR was $7.77 \%$ and $27.84 \%$, respectively. The percentage of patients with TIR $>70 \%$, TBR $<4 \%$, or TAR $<25 \%$ was $18.5 \%, 30.6 \%$, and $43.5 \%$, respectively, suggesting that targets of glycemic control were not achieved in most patients with T1D.

\section{Correlations between FGM-derived metrics and clinical variables}

As shown in Figure 1, positive correlations of HbA1c with SD, MAGE, MODD, IQR, TIR, and TAR (Spearman's $\mathrm{r}=0.69,0.49,0.34,0.43,0.46,0.56$, and 0.62 , respectively, all parameters had a $\mathrm{P}<0.01$ ) were observed. Interestingly, TG level was inversely correlated with TBR and TIR, and positively correlated with TAR (Spearman's $r=-0.34$, -0.25 , and 0.34 , respectively, $\mathrm{P}<0.01$ in all correlations). Additionally, TG level was not correlated with CV (Spearman's $\mathrm{r}=0.12, \mathrm{P}>0.05$ ), a relative measure of $\mathrm{GV}$, but was positively correlated with absolute measures of GV including SD (Spearman's $\mathrm{r}=0.24, \mathrm{P}<0.01$ ), MAGE (Spearman's $r=0.25, \mathrm{P}<0.01$ ), MODD (Spearman's $\mathrm{r}=0.20$, 
Table 1 Baseline characteristics of the study population

\begin{tabular}{|c|c|}
\hline Characteristic & Participants $(n=108)$ \\
\hline Male, n (\%) & $39(36.1 \%)$ \\
\hline Onset (year) & $27.84(21.21-38.94)$ \\
\hline Duration (years) & $7.70(3.02-13.28)$ \\
\hline Age (years) & $35.91(30.33-49.39)$ \\
\hline BMI $\left(\mathrm{kg} / \mathrm{m}^{2}\right)$ & $21.07(19.71-23.12)$ \\
\hline $\mathrm{SBP}(\mathrm{mmHg})$ & $114.00(103.00-124.00)$ \\
\hline $\mathrm{DBP}(\mathrm{mmHg})$ & $69.51 \pm 9.57$ \\
\hline WC (cm) & $76.61 \pm 8.96$ \\
\hline $\mathrm{HC}(\mathrm{cm})$ & $94.42 \pm 5.88$ \\
\hline WHR & $0.81 \pm 0.07$ \\
\hline Daily insulin dose (U/d) & $35.69 \pm 13.22$ \\
\hline Daily insulin dose (U/kg/d) & $0.60 \pm 0.20$ \\
\hline Basal insulin (U/d) & $14.25 \pm 6.71$ \\
\hline Meal insulin (U/d) & $21.44 \pm 9.32$ \\
\hline HbA1c (\%) & $7.40(6.55-8.40)$ \\
\hline $\mathrm{FPG}(\mathrm{mmol} / \mathrm{L})$ & $8.80(5.90-12.50)$ \\
\hline FCP (ng/ml) & $0.01(0.01-0.28)$ \\
\hline $\mathrm{UA}(\mathrm{mmol} / \mathrm{L})$ & $252.00(201.00-287.00)$ \\
\hline TC (mmol/L) & $4.68 \pm 1.01$ \\
\hline $\mathrm{TG}(\mathrm{mmol} / \mathrm{L})$ & $0.56(0.44-0.76)$ \\
\hline HDL-C (mmol/L) & $2.00(1.60-2.54)$ \\
\hline LDL-C (mmol/L) & $1.94(1.44-2.44)$ \\
\hline Dyslipidemia medication (\%) & $8.3 \%(9 / 108)$ \\
\hline Mean (mmol/L) & 7.92 (6.72-9.52) \\
\hline SD & $3.34 \pm 0.96$ \\
\hline CV (\%) & $40.09 \pm 7.91$ \\
\hline MODD & $3.22(2.50-3.88)$ \\
\hline MAGE & $6.87 \pm 1.77$ \\
\hline IQR (mmol/L) & $4.76 \pm 1.53$ \\
\hline TAR (\%) & $27.84(14.72-48.76)$ \\
\hline TBR (\%) & 7.77 (3.59-18.07) \\
\hline $\operatorname{TIR}(\%)$ & $58.68 \pm 16.89$ \\
\hline
\end{tabular}

BMI, body mass index; SBP, systolic blood pressure; DBP, diastolic blood pressure; WC, waist circumference; HC, hip circumference; WHR, waist-to-hip ratio; $\mathrm{HbA1c}$, hemoglobin A1c; FPG, fasting plasma glucose; FCP, fasting C-peptide; UA, uric acid; TC, total cholesterol; TG, triglyceride; HDL-C, highdensity lipoprotein cholesterol; LDL-C, low-density lipoprotein cholesterol; Mean, mean of glucose; CV, coefficient of variation for glucose; SD, standard deviation of glucose; MAGE, mean amplitude of glucose excursion; MODD, mean of daily differences; $I Q R$, interquartile range of glucose; TBR, time below range (glucose $<3.9 \mathrm{mmol} / \mathrm{L}$ ); TIR, time in range (glucose 3.9-10.0 mmol/L); TAR, time above range (glucose $>10.0 \mathrm{mmol} / \mathrm{L}$ ).
$\mathrm{P}<0.05)$, and IQR (Spearman's $\mathrm{r}=0.25, \mathrm{P}<0.01)$.

\section{Independent predictive role of TG for time in ranges determined by FGM}

To investigate the predictive role of TG for time in ranges determined by FGM, stepwise multivariate linear regression analysis was performed to adjust for potential confounders. Gender and age were adjusted in model 1. Subsequently, based on model 1, disease duration, BMI, daily insulin dose, and fasting C-peptide were further adjusted in model 2. Moreover, based on model 2, dyslipidemia medication use was further adjusted in model 3. As indicated in Table 2, TG remained significantly associated with TBR, TIR, and TAR after stepwise adjustment for potential confounders in models 1,2 , and 3, suggesting its independent predictive role in time in ranges determined by FGM among adults with T1D.

\section{Differed association between TG and TBR depending on HbA1c}

To investigate the impact of clinical variables on the relationship between TG and time in ranges derived from FGM, interaction and subgroup analyses were performed. Intriguingly, interaction was observed between HbA1c and TG in TBR (P for interaction $=0.034$ ), with subgroup analysis stratified according to $\mathrm{HbA} 1 \mathrm{c} ; \mathrm{HbA1c}<7.0 \%$ showed a borderline statistical significance ( $\mathrm{P}$ for interaction $=0.083)$, whereas no interactions were found between other clinical variables and TG in TIR and TAR ( $P$ for interaction $>0.10$ in all evaluated parameters). Furthermore, the association between TG and TBR differed substantially when patients were stratified by HbA1c. A moderate-tostrong negative correlation between TG and TBR was observed among patients with HbA1c $<7.0 \%$ ( $\mathrm{r}=-0.43$, $\mathrm{P}=0.006)$, whereas such an association was not observed among those with $\mathrm{HbA1} \mathrm{c} \geq 7.0 \%(\mathrm{r}=-0.13, \mathrm{P}=0.320)$ (Figure 2).

\section{Assessment of predictive performance of TG for the target achievement of time in ranges}

To assess the performance of TG in predicting whether the recommended targets of time in ranges (TBR $<4 \%$, TIR $>70 \%$, TAR $<25 \%$ ) could be achieved, ROC curves were established and area under the curves were calculated. Figure 3 indicated significant predictive performance 


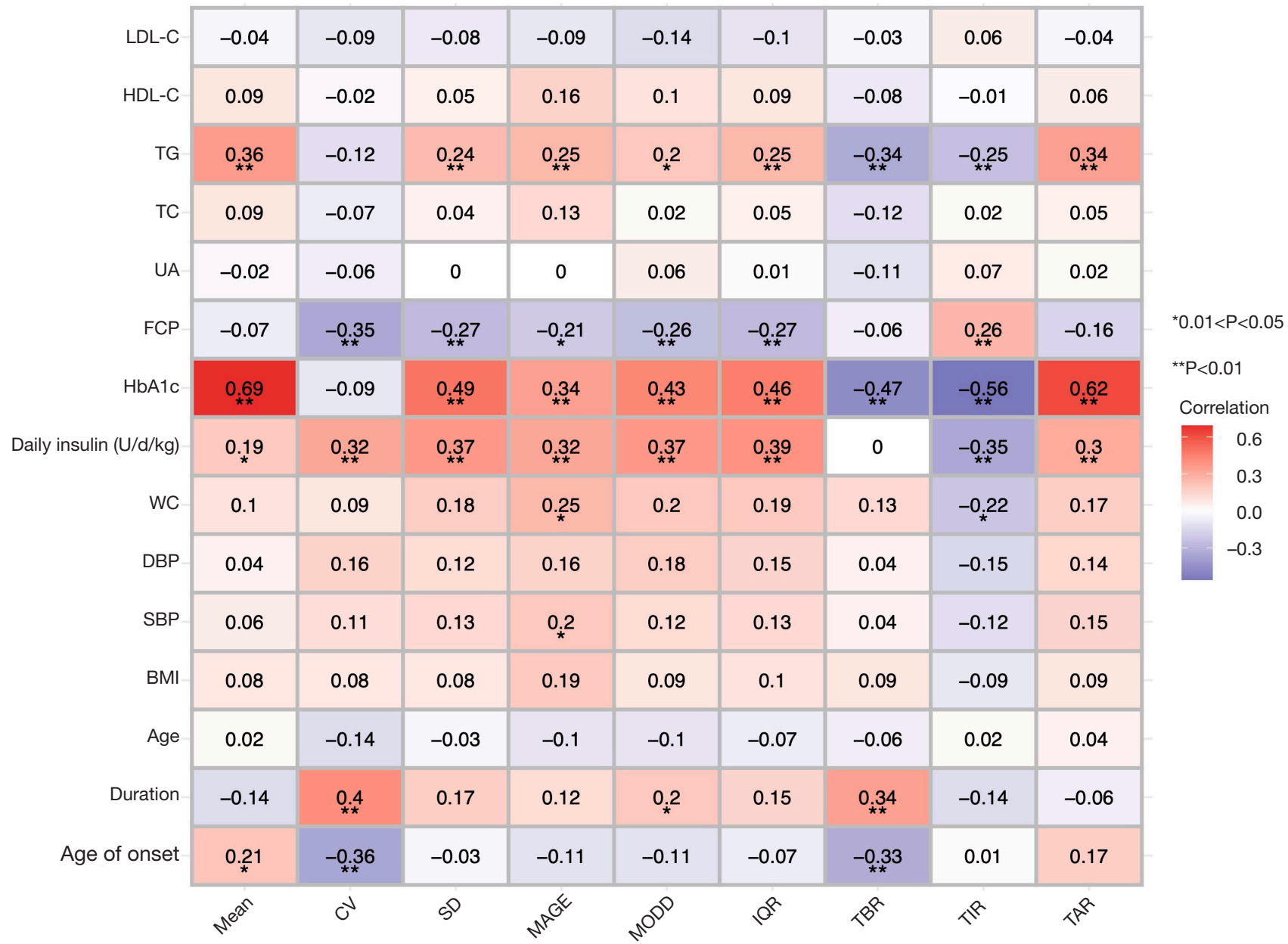

Figure 1 Spearman correlation heat map between FGM-derived metrics and clinical variables. The values of the color bar represent the Spearman's r. The red color means a positive correlation whereas the purple color means negative correlation. Darker color indicates increased correlation. ${ }^{*} \mathrm{P}<0.05 ;{ }^{* *} \mathrm{P}<0.01$. BMI, body mass index; SBP, systolic blood pressure; DBP, diastolic blood pressure; WC, waist circumference; HbA1c, hemoglobin A1c; FCP, fasting C-peptide; UA, uric acid; TC, total cholesterol; TG, triglyceride; HDL-C, highdensity lipoprotein cholesterol; LDL-C, low-density lipoprotein cholesterol; Mean, mean of glucose; CV, coefficient of variation for glucose; $\mathrm{SD}$, standard deviation of glucose; MAGE, mean amplitude of glucose excursion; MODD, mean of daily differences; IQR, interquartile range of glucose; TBR, time below range (glucose $<3.9 \mathrm{mmol} / \mathrm{L}$ ); TIR, time in range (glucose 3.9-10.0 mmol/L); TAR, time above range (glucose $>10.0 \mathrm{mmol} / \mathrm{L})$.

Table 2 Stepwise multivariate linear regression models with level of serum TG predicting time in ranges determined by FGM

\begin{tabular}{|c|c|c|c|c|c|c|}
\hline Model & \multicolumn{2}{|c|}{ TBR } & \multicolumn{2}{|c|}{ TIR } & \multicolumn{2}{|c|}{ TAR } \\
\hline Model 1 & -6.69 & 0.012 & -13.61 & 0.003 & 19.90 & $<0.001$ \\
\hline Model 2 & -7.01 & 0.006 & -13.75 & 0.002 & 20.21 & 0.001 \\
\hline Model 3 & -7.46 & 0.004 & -15.05 & $<0.001$ & 21.31 & $<0.001$ \\
\hline
\end{tabular}

Model 1: adjusted for gender and age; Model 2: adjusted for model 1 + duration + BMI + daily insulin dose + FCP; Model 3: adjusted for model 2 + dyslipidemia medication. TBR, time below range (glucose <3.9 mmol/L); TIR, time in range (glucose 3.9-10.0 mmol/L); TAR, time above range (glucose $>10.0 \mathrm{mmol} / \mathrm{L}$ ). 
Page 6 of 10

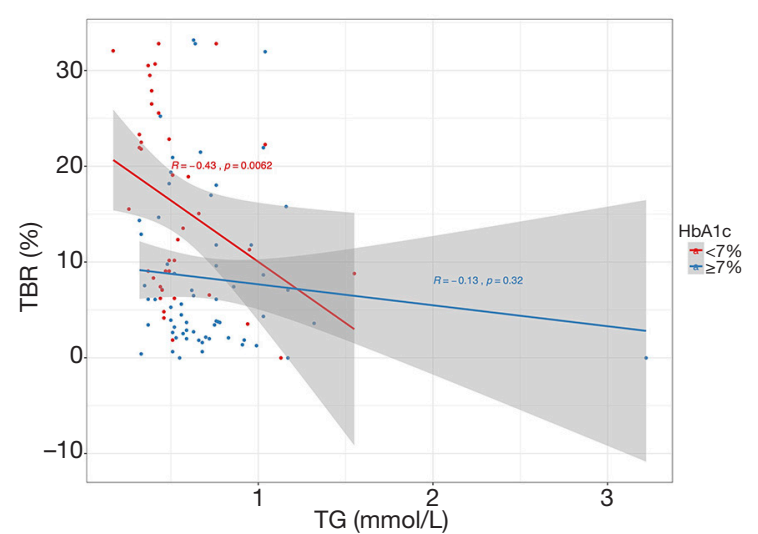

Figure 2 Scatter plots with regression line of TG and TBR. In those with HbA1c $<7.0 \%$, TG was significantly correlated with TBR (Spearman's $\mathrm{r}=-0.43, \mathrm{P}=0.006$ ). In contrast, in those with HbA1c $\geq 7.0 \%$, the association was not statistically significant (Spearman's $\mathrm{r}=-0.13, \mathrm{P}=0.320$ ). TBR, time below range (glucose $<3.9 \mathrm{mmol} / \mathrm{L})$; TG, triglyceride.

for $\mathrm{TBR}<4 \%$ (AUC $=0.704,95 \%$ CI: $0.600-0.807$, $\mathrm{P}=0.001)$ (Figure $3 A$ ) and $\mathrm{TAR}<25 \%(\mathrm{AUC}=0.693$, 95\% CI: 0.588-0.798, $\mathrm{P}=0.001$ ) (Figure 3C), whereas no significant predictive role of TG for TIR $>70 \%$ was observed (AUC $=0.576,95 \%$ CI: 0.449-0.704, P=0.296) (Figure 3D). Given that the association between TG and TBR differed substantially after stratification by HbA1c and was significant in those with $\mathrm{HbA} 1 \mathrm{c}<7.0 \%$, we further conducted ROC analysis in those with HbAlc $<7.0 \% \quad(\mathrm{n}=44)$ with an AUC 0.856 (95\% CI: 0.688-1.000, $\mathrm{P}=0.042)$ and an optimal cutoff value of TG of $0.50 \mathrm{mmol} / \mathrm{L}$ (sensitivity $100 \%$, specificity $66.7 \%$, positive predictive value $94.4 \%$ ) using Youden's index (Figure 3B).

\section{Discussion}

In the current study, we revealed, for the first time, the relationships of serum TG with measures of GV and time in range metrics derived from FGM. We found correlations between TG and absolute measures of GV, and identified the independent predictive role of TG for time in ranges determined by FGM. Further, we identified the effect of modification by HbA1c on the association between TG and TBR, indicating that serum TG testing may provide an adjunctive marker for assessment of GV and time in ranges in T1D. HbA1c may act as a potential modifier of associations between TG and TBR. It is noteworthy that modification by HbA1c could support and facilitate

\section{Liu et al. Association of triglyceride with time in hypoglycemia}

individualized therapy and decision-making for clinicians in glycemic management of T1D.

GV metrics determined by CGM could be assessed by $\mathrm{CV}$, a relative measure, and absolute measures including SD, MAGE, MODD, and IQR. CV with a recognized cutoff value of $36 \%$ has been used as a threshold to separate stable diabetes from labile diabetes in previous studies $(2,18)$. We did not find any correlation of $\mathrm{CV}$ with HbAlc or lipid profiles, whereas absolute measures of $\mathrm{GV}$ were positively correlated with HbA1c and TG. HDL-C was previously reported to correlate with CGM-derived CV in T1D, while TG level was positively correlated with CGM-derived SD in T2D without insulin therapy instead of T1D (19). This discrepancy might be explained by the small sample size of the previous study, different CGM devices, or different populations. Large-scale investigations are warranted to validate further the associations between the FGM-derived metrics and lipid profiles.

Accumulating evidence has shown that time in ranges including TIR, TAR, and TBR could be appropriate metrics of glycemic control in research and clinical settings (20). In the current study, a high TG level was independently associated with shorter TBR and TIR, and longer TAR, suggesting its potential value as an adjunctive marker of the quality of glycemic control. Previous studies demonstrated that elevated TG was closely related to chronic hyperglycemia indicated by high HbA1c in T1D $(12,13,21)$. Our findings further contributed that TG is also associated with short-term hyperglycemia detected by FGM. We found that the serum TG level was also positively correlated with a series of indicators of insulin sensitivity including BMI, waist circumference, waist to hip ratio, and HbA1c (Spearman's $r=0.22,0.22,0.31$, and 0.36 , respectively, all $\mathrm{P}<0.05)$. Thus, the possible explanation for the association of TG with TBR and TAR could be that higher serum TG level was recognized as a marker of lower insulin sensitivity $(22,23)$. Moreover, TG has been validated as one of the vital elements of insulin sensitivity clinical predictive models for T1D with favorable predictive performances $(24,25)$. TIR has been recognized as another key indicator of effective and safe glycemic control in research and clinical settings, and thus, has been validated as a reliable outcome measure in clinical trials (26) and linked to long-term diabetes complications beyond HbA1c (27). Taking these observations into consideration, it should be noted that although higher TG indicates lower risk of hypoglycemia, it suggests less time spent in the target range and higher risk of hyperglycemia. In the glycemic management 
A

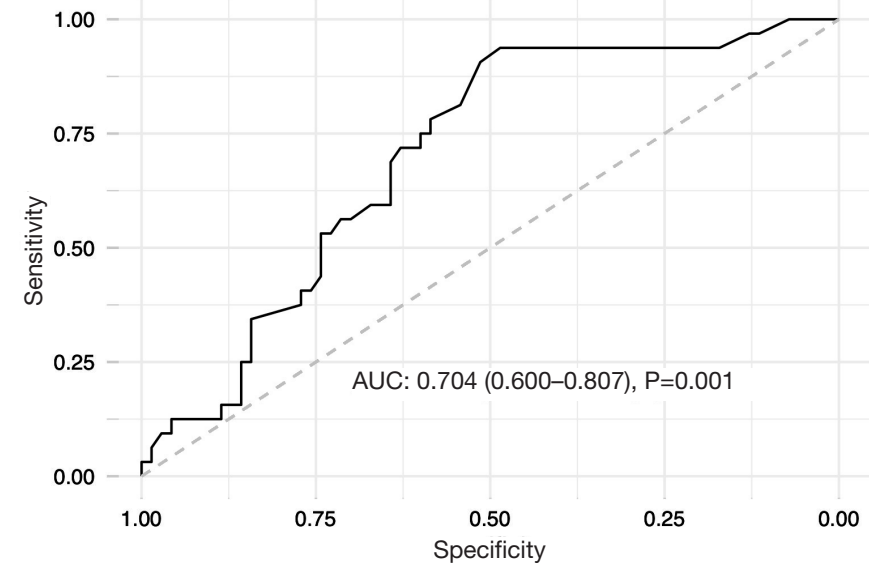

C

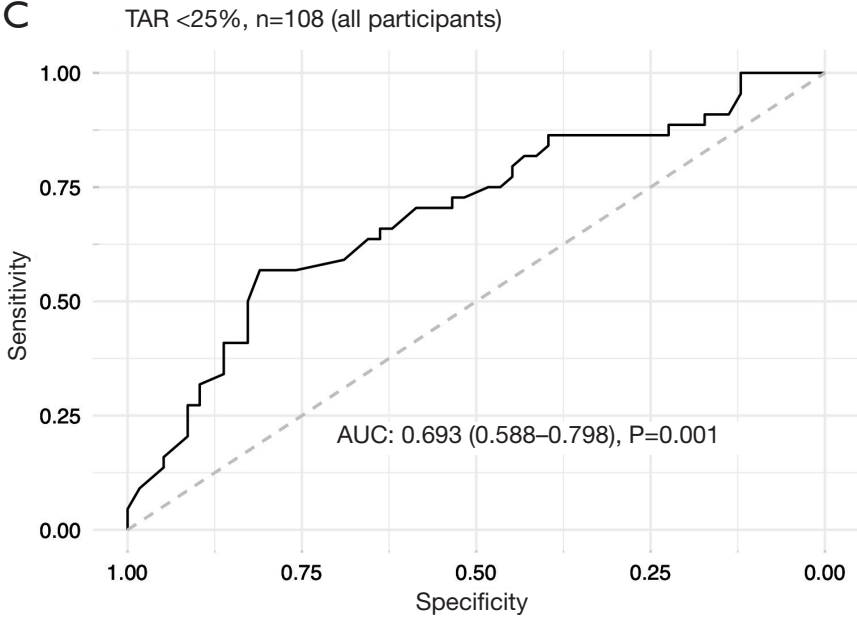

B TBR $<4 \%, n=44$ (participants with $\mathrm{HbA} 1 \mathrm{c}<7 \%$ )

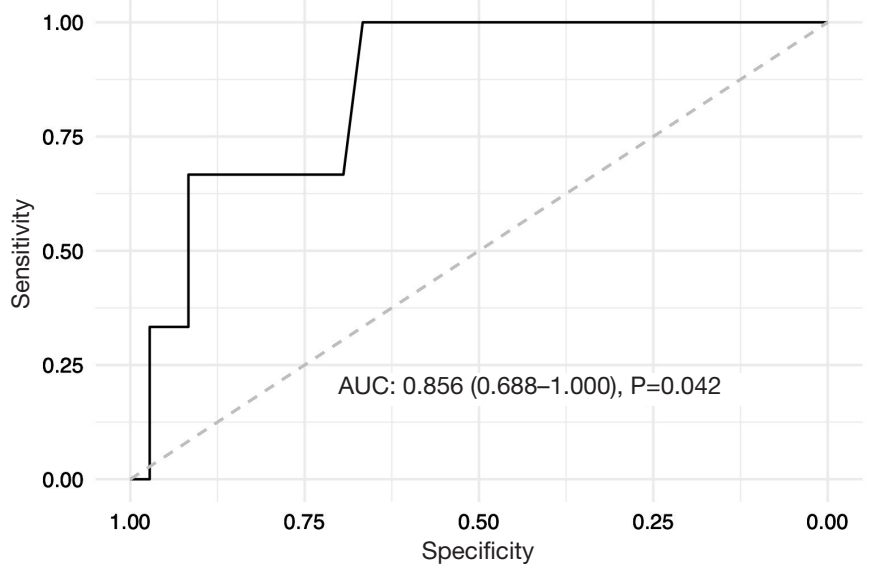

D

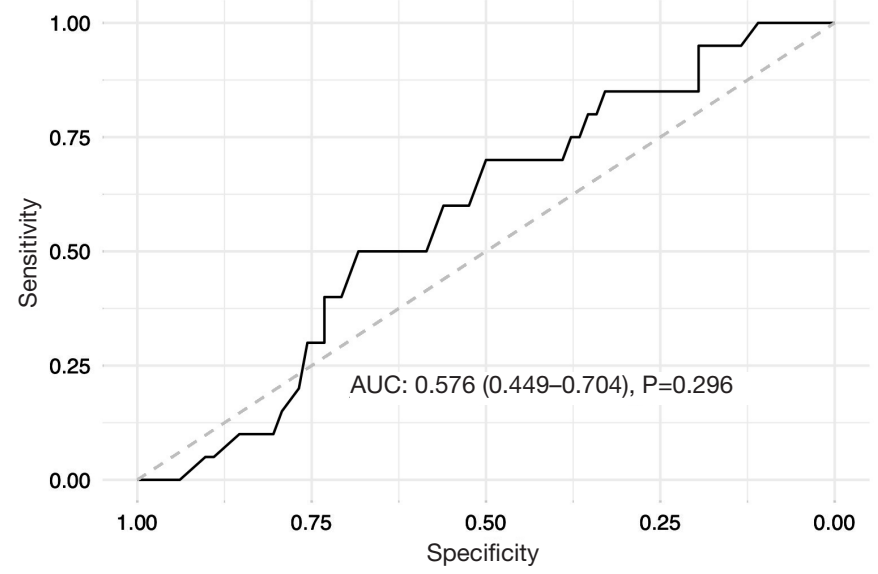

Figure 3 Predictive performance of serum TG level for targets of time in ranges using ROC curves. The area under ROC curves of TG predicting (A) TBR $<4 \%$ in all participants ( $\mathrm{n}=108$ ), (B) TBR $<4 \%$ in participants with hemoglobin A1c ( $\mathrm{n}=44$ ), (C) TAR $<25 \%$ in all participants ( $\mathrm{n}=108)$, and (D) TIR > 70\% in all participants $(\mathrm{n}=108)$ are 0.704 (95\% CI: 0.600-0.807, P=0.001), 0.856 (95\% CI: 0.688-1.000, $\mathrm{P}=0.042$ ), 0.693 (95\% CI: 0.588-0.798, P=0.001), and 0.576 (95\% CI: 0.449-0.704, P=0.296), respectively. 95\% CI, 95\% confidence intervals; ROC curves, receiver operating characteristic curves; TBR, time below range (glucose $<3.9$ mmol/L); TAR, time above range (glucose $>10.0 \mathrm{mmol} / \mathrm{L}$ ); TIR, time in range (glucose $3.9-10.0 \mathrm{mmol} / \mathrm{L}$ ).

of T1D, minimizing hypoglycemia should be the first priority, targeting $<4 \%$ of TBR, and then addressing the TIR (targeting $>70 \%$ of TIR) or hyperglycemia (targeting $<25 \%$ of TAR), as recommended by the international consensus (14). Our findings provide insights into clinical practices for clinicians in optimizing personalized medical regimen and decision-making, suggesting that adults with T1D with high TG might benefit more from adding sodium-glucose cotransporter 2 inhibitors as adjuncts to insulin, which has been demonstrated to help increase TIR and GV, without increasing TBR in T1D $(28,29)$. Additionally, given that a higher level of TG may indicate lower insulin sensitivity, for those with high TG level, metformin might be another alternative of add-on therapy to insulin because metformin has shown to improve insulin sensitivity in T1D, regardless of baseline BMI $(30,31)$. However, the results of metformin as an adjunctive therapy to insulin in T1D are contradictory. Metformin was reportedly not associated with improving overall glycemic control indicated by HbA1c or lowering TG with slight increase of severe hypoglycemia in a meta-analysis (32). Thus, although metformin could yield benefit in improving insulin sensitivity in T1D, and higher TG is associated with the lower TBR, add-on therapy of metformin in 
T1D should be prescribed with caution even in those with high TG.

Interestingly, we found an interplay between $\mathrm{HbA1c}$ and TG in the TBR determined by FGM in adults with T1D. Our data supports the predictive ability of low serum TG level for longer time spent in hypoglycemia determined by FGM, which differed substantially depending on the overall glucose control indicated by HbA1c, suggesting that HbA1c possibly modifies the association between TG and the time spent in hypoglycemia. This finding may have clinical implications, allowing clinicians to make more targeted and informed therapy decisions in glucose management of T1D. It has been previously demonstrated that lower HbAlc was associated with more frequent hypoglycemia, suggesting that improvements in glycemic control could increase the risk of hypoglycemia in T1D (33). Our observation provides additional information that is necessary for adults with T1D with good overall glycemic control and lower serum TG level to be especially more cautious about the elevated risk of hypoglycemia, and thus, a less intensive glycemic target should be set. Hence, facilitating individualized therapy regimen of diabetes management of T1D in the clinical practice.

Based on the results of ROC analysis, TG performed well in predicting T1D, achieving the recommended targets of TBR and TAR, whereas the result of TIR was disappointing. Moreover, TG performed significantly better in predicting whether the recommended target of TBR was achieved, with an optimal cutoff value of $0.50 \mathrm{mmol} / \mathrm{L}$ and a positive predictive value of $94.4 \%$, suggesting that adults with $\mathrm{HbA} 1 \mathrm{c}<7.0 \%$ accompanied by TG $<0.50 \mathrm{mmol} / \mathrm{L}$ are at high risk of time spent in hypoglycemia, longer than 1 hour per day. Therefore, it may be necessary to set a less intensive glycemic target to minimize hypoglycemia. However, the sample size of the present study was small and the cutoff point has not been validated in another population, which limits the generalizability of the finding; therefore, it should be interpreted with caution. Further investigations are needed to validate the results in the future.

Although dyslipidemia is frequently observed and has been demonstrated as an important risk factor for cardiovascular diseases in patients with T1D (34), lipid profiles within normal ranges are not unusual for individuals with T1D (35). The study population was characterized with normal levels of TG in most cases, suggesting that the TG within normal range is also beneficial for the clinical decision-making in diabetes management of T1D.
The strengths of the present study are as follows: (I) the participants were blinded to the glucose readings derived from FGM, thus avoiding any bias with respect to FGM data from the participants. (II) We are the first to explore the association of lipid profiles with FGM-derived metrics, and FGM use is rapidly increasing in routine clinical practice. However, FGM-derived metrics have been largely undefined and warrant further evaluation.

Overall, several limitations also deserve to be addressed. First, the small sample size limits the reliability of our findings when applied to clinical scenarios and thus, they should be interpreted with caution and further investigations with larger populations are warranted. Second, the absence of confirmatory blood glucose measurements in this study might also limit the reliability of the FGM readings because the glucose level and rates of glucose concentration changes affect the accuracy of FGM $(36,37)$. Finally, other confounding factors such as diet and exercise also exert significant impact on short-term GV; however, we did not collect and adjust these confounders in the current study.

\section{Conclusions}

Collectively, our findings suggest that lower serum TG levels independently predict shorter TBR and TIR, and longer TAR determined by FGM in adults with T1D. The association of TG with TBR was modified by HbA1c, which was significant in those with $\mathrm{HbA} 1 \mathrm{c}<7.0 \%$ but obscure in those with $\mathrm{HbA} 1 \mathrm{c} \geq 7.0 \%$. These data have important clinical implications for individualized therapy and decision-making in glucose management of T1D, suggesting that adults with T1D with HbA1c $<7.0 \%$ and lower serum TG levels should be more cautious about the increased risk of hypoglycemia, and thus it may be necessary to set a less intensive glycemic target. Nevertheless, further investigations are still warranted to validate these findings and the clinical value in the future.

\section{Acknowledgments}

We would like to thank Editage (www.editage.cn) for English language editing.

Funding: This work was supported by the grant from the Natural Science Foundation of Beijing Municipality (grant number 719264) and the Non-profit Central Research Institute Fund of Chinese Academy of Medical Sciences (grant number 2019XK320031). 


\section{Footnote}

Reporting Checklist: The authors have completed the STROBE reporting checklist. Available at http://dx.doi. org/10.21037/atm-20-6344

Data Sharing Statement: Available at http://dx.doi. org/10.21037/atm-20-6344

Conflicts of Interest: All authors have completed the ICMJE uniform disclosure form (available at http://dx.doi. org/10.21037/atm-20-6344). The authors have no conflicts of interest to declare.

Ethical Statement: The authors are accountable for all aspects of the work in ensuring that questions related to the accuracy or integrity of any part of the work are appropriately investigated and resolved. The study was conducted in accordance with the Declaration of Helsinki (as revised in 2013). The study was conducted under a protocol approved by the Ethics Committee of Peking Union Medical College Hospital (Approval No. B275). All participants voluntarily signed the written informed consent form.

Open Access Statement: This is an Open Access article distributed in accordance with the Creative Commons Attribution-NonCommercial-NoDerivs 4.0 International License (CC BY-NC-ND 4.0), which permits the noncommercial replication and distribution of the article with the strict proviso that no changes or edits are made and the original work is properly cited (including links to both the formal publication through the relevant DOI and the license). See: https://creativecommons.org/licenses/by-nc-nd/4.0/.

\section{References}

1. Zinman B, Marso SP, Poulter NR, et al. Day-to-day fasting glycaemic variability in DEVOTE: associations with severe hypoglycaemia and cardiovascular outcomes (DEVOTE 2). Diabetologia 2018;61:48-57.

2. Danne T, Nimri R, Battelino T, et al. International Consensus on Use of Continuous Glucose Monitoring. Diabetes Care 2017;40:1631-40.

3. Beck RW, Connor CG, Mullen DM, et al. The Fallacy of Average: How Using HbA1c Alone to Assess Glycemic Control Can Be Misleading. Diabetes Care 2017;40:994-9.

4. Riddlesworth TD, Beck RW, Gal RL, et al. Optimal
Sampling Duration for Continuous Glucose Monitoring to Determine Long-Term Glycemic Control. Diabetes Technol Ther 2018;20:314-6.

5. Beyond ACWG. Need for Regulatory Change to Incorporate Beyond A1C Glycemic Metrics. Diabetes Care 2018;41:e92-4.

6. Chehregosha H, Khamseh ME, Malek M, et al. A View Beyond HbA1c: Role of Continuous Glucose Monitoring. Diabetes Ther 2019;10:853-63.

7. Ang E, Lee ZX, Moore S, et al. Flash glucose monitoring (FGM): A clinical review on glycaemic outcomes and impact on quality of life. J Diabetes Complications 2020;34:107559.

8. Castellana M, Parisi C, Di Molfetta S, et al. Efficacy and safety of flash glucose monitoring in patients with type 1 and type 2 diabetes: a systematic review and meta-analysis. BMJ Open Diabetes Res Care 2020;8:e001092.

9. Gomez-Peralta F, Dunn T, Landuyt K, et al. Flash glucose monitoring reduces glycemic variability and hypoglycemia: real-world data from Spain. BMJ Open Diabetes Res Care 2020;8:e01052.

10. Mitsuishi S, Nishimura R, Harashima SI, et al. The Effect of Novel Glucose Monitoring System (Flash Glucose Monitoring) on Mental Well-being and Treatment Satisfaction in Japanese People with Diabetes. Adv Ther 2018;35:72-80.

11. Yaron M, Roitman E, Aharon-Hananel G, et al. Effect of Flash Glucose Monitoring Technology on Glycemic Control and Treatment Satisfaction in Patients With Type 2 Diabetes. Diabetes Care 2019;42:1178-84.

12. Maahs DM, Dabelea D, D'Agostino RB Jr, et al. Glucose control predicts 2-year change in lipid profile in youth with type 1 diabetes. J Pediatr 2013;162:101-7.e1.

13. Maahs DM, Ogden LG, Dabelea D, et al. Association of glycaemia with lipids in adults with type 1 diabetes: modification by dyslipidaemia medication. Diabetologia 2010;53:2518-25.

14. Battelino T, Danne T, Bergenstal RM, et al. Clinical Targets for Continuous Glucose Monitoring Data Interpretation: Recommendations From the International Consensus on Time in Range. Diabetes Care 2019;42:1593-603.

15. Monnier L, Wojtusciszyn A, Molinari N, et al. Respective Contributions of Glycemic Variability and Mean Daily Glucose as Predictors of Hypoglycemia in Type 1 Diabetes: Are They Equivalent? Diabetes Care 2020;43:821-7.

16. Service FJ, Molnar GD, Rosevear JW, et al. Mean 
amplitude of glycemic excursions, a measure of diabetic instability. Diabetes 1970;19:644-55.

17. Molnar GD, Taylor WF, Ho MM. Day-to-day variation of continuously monitored glycaemia: a further measure of diabetic instability. Diabetologia 1972;8:342-8.

18. Monnier L, Colette C, Wojtusciszyn A, et al. Toward Defining the Threshold Between Low and High Glucose Variability in Diabetes. Diabetes Care 2017;40:832-8.

19. Jin SM, Kim TH, Bae JC, et al. Clinical factors associated with absolute and relative measures of glycemic variability determined by continuous glucose monitoring: an analysis of 480 subjects. Diabetes Res Clin Pract 2014;104:266-72.

20. Rodbard D. Glucose Time In Range, Time Above Range, and Time Below Range Depend on Mean or Median Glucose or HbA1c, Glucose Coefficient of Variation, and Shape of the Glucose Distribution. Diabetes Technol Ther 2020;22:492-500.

21. Reh CM, Mittelman SD, Wee CP, et al. A longitudinal assessment of lipids in youth with type 1 diabetes. Pediatr Diabetes 2011;12:365-71.

22. Uruska A, Zozulinska-Ziolkiewicz D, Niedzwiecki P, et al. TG/HDL-C ratio and visceral adiposity index may be useful in assessment of insulin resistance in adults with type 1 diabetes in clinical practice. J Clin Lipidol 2018;12:734-40.

23. Maahs DM, Nadeau K, Snell-Bergeon JK, et al. Association of insulin sensitivity to lipids across the lifespan in people with Type 1 diabetes. Diabet Med 2011;28:148-55.

24. Dabelea D, D'Agostino RB, Jr., Mason CC, et al. Development, validation and use of an insulin sensitivity score in youths with diabetes: the SEARCH for Diabetes in Youth study. Diabetologia 2011;54:78-86.

25. Duca LM, Maahs DM, Schauer IE, et al. Development and Validation of a Method to Estimate Insulin Sensitivity in Patients With and Without Type 1 Diabetes. J Clin Endocrinol Metab 2016;101:686-95.

26. Beck RW, Bergenstal RM, Riddlesworth TD, et al. Validation of Time in Range as an Outcome Measure for Diabetes Clinical Trials. Diabetes Care 2019;42:400-5.

27. Lu J, Ma X, Zhou J, et al. Association of Time in Range, as Assessed by Continuous Glucose Monitoring, With Diabetic Retinopathy in Type 2 Diabetes. Diabetes Care 2018;41:2370-6.

28. Mathieu C, Dandona P, Phillip M, et al. Glucose Variables in Type 1 Diabetes Studies With Dapagliflozin: Pooled Analysis of Continuous Glucose Monitoring Data From DEPICT-1 and -2. Diabetes Care 2019;42:1081-7.
29. Famulla S, Pieber TR, Eilbracht J, et al. Glucose Exposure and Variability with Empagliflozin as Adjunct to Insulin in Patients with Type 1 Diabetes: Continuous Glucose Monitoring Data from a 4-Week, Randomized, PlaceboControlled Trial (EASE-1). Diabetes Technol Ther 2017;19:49-60.

30. Cree-Green M, Bergman BC, Cengiz E, et al. Metformin Improves Peripheral Insulin Sensitivity in Youth With Type 1 Diabetes. J Clin Endocrinol Metab 2019;104:3265-78.

31. Bjornstad P, Schafer M, Truong U, et al. Metformin Improves Insulin Sensitivity and Vascular Health in Youth With Type 1 Diabetes Mellitus. Circulation 2018;138:2895-907.

32. Meng H, Zhang A, Liang Y, et al. Effect of metformin on glycaemic control in patients with type 1 diabetes: A metaanalysis of randomized controlled trials. Diabetes Metab Res Rev 2018;34:e2983.

33. Philis-Tsimikas A, Lane W, Pedersen-Bjergaard U, et al. The relationship between HbA1c and hypoglycaemia in patients with diabetes treated with insulin degludec versus insulin glargine 100 units/mL. Diabetes Obes Metab 2020;22:779-87.

34. Morrish NJ, Wang SL, Stevens LK, et al. Mortality and causes of death in the WHO Multinational Study of Vascular Disease in Diabetes. Diabetologia 2001;44 Suppl 2:S14-21.

35. Feitosa AC, Feitosa-Filho GS, Freitas FR, et al. Lipoprotein metabolism in patients with type 1 diabetes under intensive insulin treatment. Lipids Health Dis 2013;12:15.

36. Moser O, Eckstein ML, McCarthy O, et al. Performance of the Freestyle Libre flash glucose monitoring (flash GM) system in individuals with type 1 diabetes: A secondary outcome analysis of a randomized crossover trial. Diabetes Obes Metab 2019;21:2505-12.

37. Cao B, Wang R, Gong C, et al. An Evaluation of the Accuracy of a Flash Glucose Monitoring System in Children with Diabetes in comparison with Venous Blood Glucose. J Diabetes Res 2019;2019:4845729.

Cite this article as: Liu Y, Yu J, Ma C, He S, Ping F, Zhang H, Li W, Xu L, Xiao X, Li Y. Hemoglobin Alc modifies the association between triglyceride and time in hypoglycemia determined by flash glucose monitoring in adults with type 1 diabetes: implications for individualized therapy and decisionmaking. Ann Transl Med 2021;9(7):537. doi: 10.21037/atm-206344 